\title{
Test yourself answer to question: atraumatic fracture of an abnormal humerus
}

\author{
Mostafa Ellatif $^{1}\left(\mathbb{D} \cdot \operatorname{lan}\right.$ Pressney $^{1} \cdot$ Daniel Lindsay $^{2} \cdot$ Paul O'Donnell $^{1}$
}

Received: 6 September 2021 / Revised: 5 October 2021 / Accepted: 8 October 2021 / Published online: 16 October 2021

(c) ISS 2021

\section{Diagnosis}

Hydatid disease of bone

\section{Discussion}

Radiographs of the humerus demonstrate a minimally expansile, lytic, intramedullary lesion involving nearly the entire bone, with internal septations, endosteal scalloping and an oblique pathological fracture (Fig. 1a). There is minor disease progression distally and fracture non-union at 12-month follow-up. Widening of the subacromial space was not explained by subsequent MRI, which excluded articular or bursal involvement, and may be partly projectional (Fig. 1b). $\mathrm{CT}$ of the humerus confirms radiographic appearances without intrinsic 'double layered' mineralisation, 'rind' sign or ground glass attenuation (Fig. 2). MRI demonstrates extensive multi-locular cystic spaces at presentation (Fig. 3a) with follow-up MRI at 12 months suggesting progressive disease: collapse of the diaphyseal cystic spaces (Fig. 3b) and development of an extra-osseous lesion with similar imaging features. Axial MRI of the latter shows peripherally sited 'daughter' cysts and a 'floating membrane' sign (Fig. 4), and on ultrasound there is an anechoic cystic lesion with well-defined capsule and echogenic, layering material lying dependently, suggestive of a detached laminated membrane (the 'water lily' sign of hydatid disease [HD]) (Fig. 5). Histopathology showed the typical features of HD,

The case presentation can be found at doi: $10.1007 / \mathrm{s} 00256-021-$ 03,940-3

Mostafa Ellatif

Mostafa.ellatif@nhs.net

1 Department of Radiology, Royal National Orthopaedic Hospital, Stanmore, UK

2 Department of Histopathology, Royal National Orthopaedic Hospital, Stanmore, UK with a protoscolex of Echinococcus granulosus, which is composed of a rostellum (Fig. 6a) (hooks for attachment, black arrow) - the sucker cups are not clearly visible in this plane of section. Figure $6 \mathrm{~b}$ shows the collapsed wall of an Echinococcus granulosus cyst, which would normally contain the protoscolices. The germinal epithelium is also clearly visible.

HD is a zoonosis commonly caused by Echinococcus granulosus tapeworm and is endemic in North Africa and the Middle East [1], with infection acquired faeco-orally through ingestion of the larvae. Bone infection is uncommon (0.5-2\% of cases) and classically affects the spine, pelvis, femora and tibiae, with humeral involvement rare [2]. Our patient had no history of travel to endemic areas.

Patients with osseous HD are typically asymptomatic and usually present due to a complication such as pathological fracture. The hydatid cyst has three layers: the outer pericyst, the middle ectocyst and the inner germinal layer. The two innermost layers together form the endocyst [3]. In bone, the pericyst does not form: embryos are deposited directly and the daughter vesicles gradually replace bone marrow. On radiographs, stage I osseous disease shows a moth-eaten appearance/ill-defined epimetaphyseal lucency. In stage II, there are large cystic areas with cortical bone loss; in stage III, there is breach of the cortex and extra-osseous extension.

On CT, a rounded cystic lesion with double layered calcification is specific for HD [4]. MRI is the imaging modality of choice, demonstrating cystic lesions which show low to intermediate signal on T1W sequences and hyperintense T2W/STIR signal. Parent cysts may appear hyperintense to daughter cysts on T1W images [4]. In a contained rupture, the endocyst can separate from the pericyst resulting in a 'floating membrane' or 'water lily' sign, as demonstrated in our case (Figs. 4 and 5). Hydatid cysts are avascular and hence show no central enhancement but may show peripheral enhancement [5]. Ultrasound is the imaging modality of choice in non-osseous HD, demonstrating anechoic cystic 

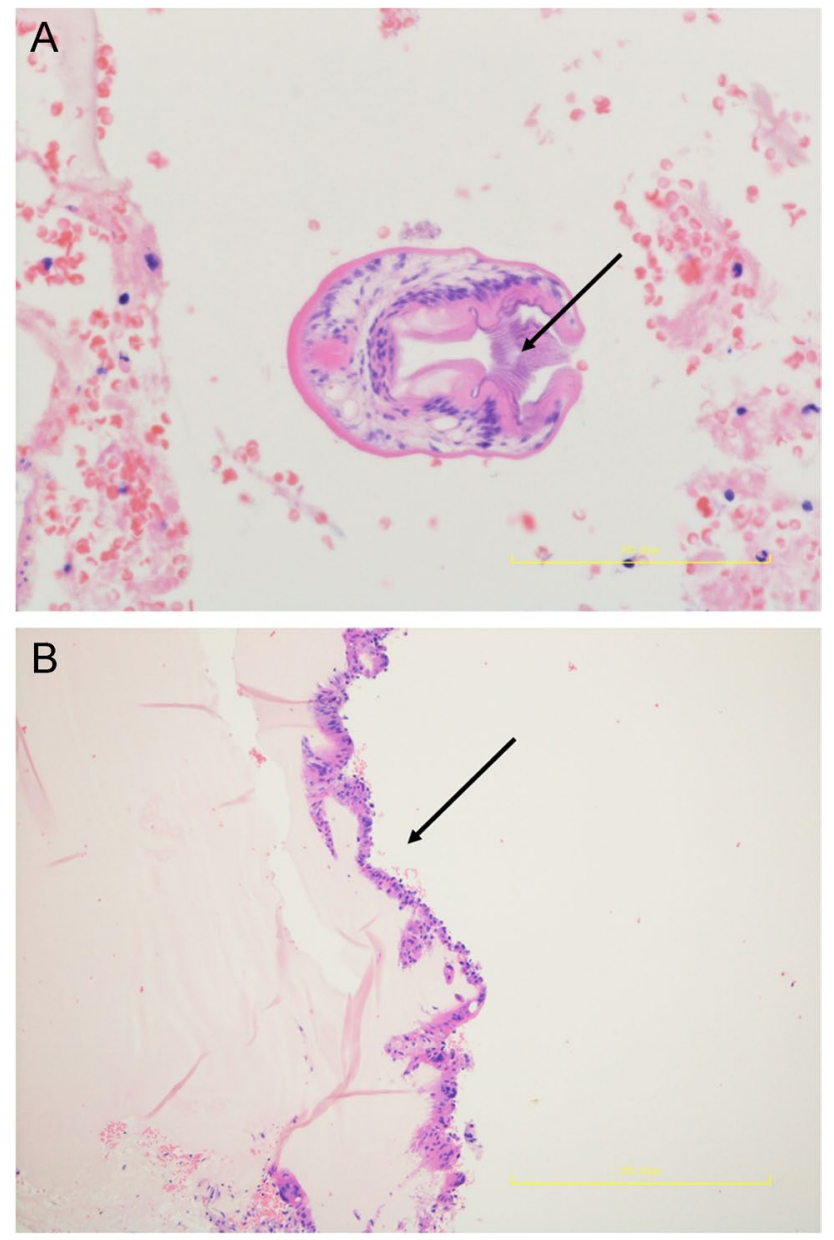

Fig. 6 a (with annotation): (H\&E, $\times 40)$ : Intraosseous hydatid disease. This section shows a protoscolex of Echinococcus granulosus, which is composed of a rostellum (hooks for attachment, black arrow). b (with annotation): $(\mathrm{H} \& \mathrm{E}, \times 10)$ : Intraosseous hydatid disease. This section shows the collapsed wall of an Echinococcus granulosus cyst with the germinal epithelium also clearly visible (black arrow)

lesions with a floating membrane, the pathognomonic 'water lily' sign [6].

Differential diagnosis on imaging includes cystic fibrous dysplasia, tuberculosis and chronic osteomyelitis. Our patient was initially thought to have fibrous dysplasia with no evidence of polyostotic disease on whole-body MRI; blood markers for infection were normal. The diagnosis was made surgically, following progression of the imaging features, persistent symptoms and failure of US-guided aspiration of the cystic component and core biopsy targeting the peripheral more solid components (Fig. 5), with no convincing evidence of delaminated membrane in the retrieved samples. This highlights the difficulty in making a definitive diagnosis. Serological assessment may be helpful, but only has a sensitivity of approximately $60 \%$ in osseous HD [7]. Treatment typically involves wide surgical excision and a systemic anti-helminthic agent such as albendazole [8], although documented surgical treatment of osseous HD is limited. Currently, our patient is undergoing medical treatment with a view to endo-prosthetic replacement for symptom relief.

The identification of multilocular cysts, and the other features described above, in the bone of a relatively asymptomatic patient should alert the radiologist to the possibility of rare osseous HD.

\section{Declarations}

Conflict of interest The authors declare no competing interests.

\section{References}

1. Zalaquett E, Menias C, Garrido F, Vargas M, Olivares JF, Campos $\mathrm{D}$, et al. Imaging of hydatid disease with a focus on extrahepatic involvement. Radiogr Rev Publ Radiol Soc N Am Inc. 2017: 37(3):901-23.

2. Pedrosa I, Saíz A, Arrazola J, Ferreirós J, Pedrosa CS. Hydatid disease: radiologic and pathologic features and complications. Radiogr Rev Publ Radiol Soc N Am Inc. 2000;20(3):795-817.

3. Paruchuri RK, Kapoor A. Osseous hydatid disease - a series of cases. Indian J Musculoskelet Radiol. 2020;2:120-4.

4. Song XH, Ding LW, Wen H. Bone hydatid disease. Postgrad Med J. 2007;83(982):536-42.

5. Bermejo A, De Bustamante TD, Martinez A, Carrera R, Zabía E, Manjón P. MR imaging in the evaluation of cystic-appearing soft-tissue masses of the extremities. Radiogr Rev Publ Radiol Soc N Am Inc. 2013; 33(3):833-55.

6. Mehta P, Prakash M, Khandelwal N. Radiological manifestations of hydatid disease and its complications. Trop Parasitol. 2016;6(2):103-12.

7. Monge-Maillo B, Olmedo Samperio M, Pérez-Molina JA, Norman F, Mejía CR, Tojeiro SC, et al. Osseous cystic echinococcosis: a case series study at a referral unit in Spain. Tamarozzi F, editor. PLoS Negl Trop Dis. 2019;13(2):e0007006.

8. Dziri C, Haouet K, Fingerhut A, Zaouche A. Management of cystic echinococcosis complications and dissemination: where is the evidence? World J Surg. 2009;33(6):1266-73.

9. Zlitni M, Ezzaouia K, Lebib H, Karray M, Kooli M, Mestiri M. Hydatid cyst of bone: diagnosis and treatment. World J Surg. 2001;25(1):75-82.

Publisher's note Springer Nature remains neutral with regard to jurisdictional claims in published maps and institutional affiliations. 